\title{
The safety and tolerance of phytotherapies in menopausal medicine - a review of the literature
}

\author{
Piotr Czuczwar, Tomasz Paszkowski, Marek Lisiecki, Sławomir Woźniak, Anna Stępniak \\ $3^{\text {rd }}$ Chair and Department of Gynecology, Medical University of Lublin, Poland
}

\begin{abstract}
Phytoestrogens are polyphenol, non-steroidal substances of plant origin, resembling $17 \beta$-estradiol in structure. These substances can act as either agonists or antagonists of oestrogen receptors $\alpha$ and $\beta$. Phytoestrogens are widely used to alleviate menopausal symptoms, such as hot flushes and night sweats. Most of the currently available products of plant origin registered to soften climacteric symptoms consist of extracts obtained from soy, red clover, or black cohosh.

Non-hormonal phytotherapy is a new alternative for patients suffering from menopausal symptoms. Active ingredients such as PI 82-GC FEM extract do not show any direct hormonal mechanisms of action typical for oestrogens and phytoestrogens.

There are concerns about the safety and tolerability of phytoestrogens. In this review we summarise the current literature regarding the clinical aspect of safety and tolerance of different phytotherapies used to relieve menopausal symptoms.
\end{abstract}

Key words: phytoestrogens, menopause, phytotherapy.

\section{Introduction}

\section{Phytoestrogens}

Phytoestrogens are polyphenol, non-steroidal substances of plant origin, resembling $17 \beta$-estradiol in structure. These substances can act as either agonists or antagonists of oestrogen receptors $\alpha$ and $\beta$ (ER $\alpha$ and $\mathrm{ER} \beta)[1,2]$.

The affinity of phytoestrogens is considered to be sevenfold greater to $E R \beta$ than to $E R \alpha[3,4]$. The biological activity of isoflavones on ERs depends on the level of endogenous oestrogens: at high levels of endogenous oestrogens the isoflavones exert antagonistic properties, while at low levels they act as ER agonists [3].

Phytoestrogens are divided into three major classes:

- Lignans (secoisolariciresinol and matairesinol)

- Stilbene (resveratrol: found in the skin of many fruits, inter alia grapes and wines)

- Flavonoids (isoflavones, which include four subgroups: isoflavones, isoflavanones, isoflavanes, and coumestans)

Most of the available studies focus on three phytohormones: isoflavones, lignans, and coumestans. These compounds can be found in different plants, their biological activity also varies:

- Isoflavones - present mainly in soy, lens, chickpea, beans, broad beans, hops, and cereals, including wheat, barley, and rye.
- Lignans - found in seed oils (mainly linseed oil), but they are also present in sunflower seeds, linseeds, garlic, onion, cherries, pears, apples, hop beer, and red wine.

- Kumen derivatives (coumestans) - not present in the ingredients of standard diets. These compounds can be found mainly in red clover and, in lower concentrations, in sunflower seeds and soy sprouts.

The richest sources of isoflavones (genistein, daidzein, formononetin, biochanin A, glycitein) are mainly leguminous vegetables (soy and its preserves, leguminous vegetables seeds, lentil, spinach, and red clover).

\section{Non-hormonal phytotherapy}

Non-hormonal phytotherapy is a new therapeutic alternative for patients suffering from menopausal symptoms. The most promising preparation is a dietary supplement composed of standardised extracts from pollen and pistil (PI 82-GC FEM) combined with Vitamin $\mathrm{E}$ as an antioxidant.

The PI 82 extract is obtained from pollen of $\mathrm{Se}$ cale cereale, Dactylis glomerata, and Pinus silvestris. PI 82 also consists of extracts from Zea mays pollen and pistil. The other active compound included in Femelis Meno ${ }^{\circledR}$ is GC FEM, which is an extract from Secale cereale, Zea mays, and Pinus silvestris pollen. Pollen and 
pistil are selected and collected separately using a standardised method. Cultivation of selected plant species is led under strict supervision, similarly to the process of extraction of active substances. This product was introduced to the Swedish market in 1995 and is now available in 15 countries, including Poland. The efficacy of this preparation in decreasing vasomotor menopaus al symptoms was discovered accidentally during a clinical study evaluating its generally strengthening effect in elderly patients [5].

The PI 82-GC FEM extracts do not show any direct hormonal mechanisms of action typical for oestrogens and phytoestrogens. It is suggested that PI 82-GC FEM extracts act similarly to SSRIs by modulating the activity of serotonergic neurons in the central nervous system, which control thermoregulation, mood, and sleep. It was found that PI 82-GC FEM extracts obtained from pollen and pistil inhibit serotonin uptake in rats' cortical synaptosomes [5].

\section{Tolerability and safety of phytoestrogens}

The safety and tolerance of long-term supplementation with phytoestrogens of soy origin and isoflavones from black cohosh and red clover remain controversial.

\section{Red clover}

A study comparing the occurrence of adverse reactions in women using red clover extract and placebo did not show any significant differences (RR 0.95, 95\% $\mathrm{Cl}: 0.65$ to 1.40) [6]. Moreover, no statistical differences were found in the occurrence of respiratory tract infections, headaches, muscle aches, sicknesses, joint pains, diarrhoeas, and abnormal uterine bleeding.

Three studies investigated the possible impact of red clover on endometrial thickness [7-9]. Baber et al. did not observe significant differences in endometrial thickness after 12 weeks of red clover treatment. Interestingly, Imhof et al. demonstrated a significant decrease in endometrial thickness (by approximately $15 \%)$ after three months of red clover treatment in comparison to placebo $(p<0.001)$ [7]. No differences in endometrial thickness were observed in this study among patients using red clover or placebo [8]

\section{Soy}

Among the six studies evaluating the frequency of adverse reactions in women consuming meals rich in soy, five did not reveal any significant alterations in comparison to the subjects on soy-poor diet. However, in one study $75 \%$ of women using the soy-rich diet reported bloating, sickness, body weight gain, and gastrointestinal disturbances, in contrast to the placebo group, in which these symptoms occurred in only $17 \%$ of women [10].

Most of the studies investigating the safety of soy extracts did not show any influence of soy extracts on endometrial thickness, vaginal maturation index, and occurrence of adverse reactions in comparison to placebo. Kaari et al. observed a significant improvement in vaginal $\mathrm{pH}$ and vaginal maturity index in patients using oestrogen therapy in comparison to those using soy extract [11]. On the other hand, soy extracts produced less endometrial stimulation and fewer adverse reactions (such as uterine bleeding). Another study showed that soy extract has a positive effect on vaginal $\mathrm{pH}$ in comparison to placebo [12].

One study demonstrated a significant increase in the percentage of constipation and fractures in patients using soy extracts in comparison to placebo [13]. However, the authors concluded that the increased incidence of fractures was not directly related to the treatment option. Fritz et al. suggested that soy consumption may result in a decrease of breast cancer morbidity, recurrence, and mortality [14].

Three of the available studies reported a significant increase in the occurrence of adverse reactions in patients treated with soy extracts $[10,13,15]$. Soy extract powder resulted in taste aversion, which probably resulted from the product type [10]. Levis et al. evaluated the influence of soy on bone mass loss and vasomotor symptoms [13]. Surprisingly, the incidence of fractures was significantly higher in the group of women taking soy diet supplements.

The results of a few publications suggest that a cancerogenic action of long-term soya isoflavones cannot be excluded. Shike et al. showed that exposure to soy genistein enhances the gene expression in the MCF7 cells of breast cancer, which may initiate the cancerogenesis [16]. De Lemos published a meta-analysis of studies published between 1966 and 2001, which revealed that soy origin genistein and daidzein even in low concentrations can stimulate the growth of malignant breast tumours in both an in vitro model and in animal experiments. Moreover, these isoflavones were shown to weaken the antineoplastic action of tamoxifen. In conclusion of this meta-analysis, caution is recommended in using soya isoflavones in patients during or after breast cancer treatment [17].

\section{Black cohosh}

\section{Black cohosh vs. placebo}

Several studies investigating adverse reactions to black cohosh have been published [18-23]. In a group of 430 women treated with black cohosh a total of 194 adverse reactions were observed (0.45 per patient), while in the placebo group (392 patients) - 195 (0.50 
per patient). No significant differences in the incidence of adverse reactions were found in the group of women using black cohosh.

\section{Black cohosh vs. hormonal therapy}

Four studies analysed the safety profile of black cohosh in comparison to hormonal therapy [19, 23-25]. In the group of 253 women taking black cohosh a total of 202 adverse reactions were noted ( 0.80 per person) in comparison to 304 reactions among 208 women using hormonal therapy (1.46 per person). However, this difference was not statistically significant.

In terms of safety, black cohosh was also compared to red clover and fluoxetine - these isolated reports do not allow firm conclusions to be drawn.

A review of literature concerning black cohosh safety included a total of 4232 women. Adverse reactions due to the use of black cohosh (6.5 to $160 \mathrm{mg}$ ) with a follow-up ranging from one to twelve months were reported. The observed adverse reactions were relatively rare, mild, and transient. The most commonly reported adverse effects were related to the gastrointestinal system, musculoskeletal system, and connective tissue [26]. Individual cases of hepatitis, hepatic insufficiency, facial oedema, and cutaneous vasculitis were also noted, but there is no evidence of a causal relationship between these adverse reactions and black cohosh [27].

\section{Tolerance and safety of non-hormonal phytotherapy}

One of the main concerns in the context of PI 82-GC FEM extract safety profile is the possibility of allergic reactions due to the strong allergic properties of pollen. However, during the extraction process purified cytoplasm of pollen cells is obtained by removing the coat, which consists mainly of allergens. The allergens that are present in pollen are completely removed, which is why this product may also be used in patients with various allergies. After such processing pollen cytoplasm consists almost completely of bioactive substances that have a very high bioavailability following oral intake.

The safety profile of PI 82-GC FEM extracts has been evaluated by several studies. Elia and Mares examined 417 women suffering from menopausal symptoms (average age: 54.4 years), mainly of vasomotor origin. These patients were treated with a drug containing 40 $\mathrm{mg}$ of GC FEM extract, $120 \mathrm{mg}$ of PI 82 extract, and $5 \mathrm{mg}$ of Vitamin $\mathrm{E}$ (two pills per day) for 84 days. More than $98 \%$ of the study participants reported the tolerance of this therapy to have been very good [28].

Winther et al. conducted a randomised, placebo-controlled study in which the efficacy of PI 82-GC FEM extracts in decreasing menopausal symptoms (mainly hot flushes) was evaluated. Fifty-four women randomised to three-month treatment with PI 82-GC FEM extracts or placebo were included in the study. Only minor and clinically insignificant adverse events were noted, and the tolerance profile of this product was evaluated to be very good [29].

Kimura and Gruber investigated a product containing PI 82-GC FEM extracts and confirmed its efficacy in reducing menopausal symptoms, such as hot flushes (average reduction of 57.3\%), night sweats (average reduction of $62.6 \%$ ), and sleep disorders (average reduction of $54.7 \%)$. No adverse events were found in this study, including allergic reactions [30].

Purified Swedish pollen extract seems a very interesting treatment option in breast cancer patients treated with tamoxifen. Tamoxifen treatment may result in the onset or exacerbation of vasomotor symptoms. However, such patients should not receive systemic oestrogens or phytoestrogens because these compounds may have a proliferative effect on breast cancer cells. Currently, selective serotonin reuptake inhibitors (SSRIs) are approved for treatment of tamoxifen-induced vasomotor symptoms. Unfortunately, SSRIs may interfere with the efficacy of tamoxifen by inhibiting CYP2D6, a member of the cytochrome P450 enzyme family, which is crucial in the metabolism of tamoxifen [31]. Interestingly, in an in vitro study Swedish pollen extract did not inhibit CYP2D6 [32]. Taking into consideration that Swedish pollen does not act by hormonal mechanisms and does not inhibit the activity of CYP2D6, it seems a reasonable option for the prevention and treatment of vasomotor symptoms in breast cancer patients receiving tamoxifen.

The Polish Menopause and Andropause Society (PMAS) recently published a statement concerning a product containing PI 82-GC FEM extracts. According to the PMAS Expert Team statement, available literature provides evidence that the efficacy and safety profile of PI 82-GC FEM extracts used in order to reduce menopausal symptoms is favourable [5].

\section{Conclusions}

Current literature concerning the safety and tolerance profile of phytoestrogens provides equivocal results - further studies are required to provide firm evidence, especially regarding the use of soya isoflavones in breast cancer survivors. A non-hormonal phytotherapeutic option should be taken into consideration when treating patients with vasomotor menopausal symptoms, especially in cases where the safety of the therapy is of special importance.

\section{Disclosure}

Authors report no conflict of interests. 


\section{References}

1. Kuiper GG, Lemmen JG, Carlsson B, et al. Interaction of estrogenic chem icals and phytoestrogens with estrogen receptor beta. Endocrinology 1998; 139: 4252-4263.

2. Miksicek RJ. Interaction of naturally occurring nonsteroidal estrogens with expressed recombinant human estrogen receptor. J Steroid Biochem Mol Biol 1994; 49: 153-160.

3. Setchell KD, Brown NM, Lydeking-Olsen E. The clinical importance of the metabolite equol - a clue to the effectiveness of soy and its isofalvones. J Nutr 2002; 132: 3577-3584.

4. Panay N. Taking an integrated approach: managing women with phytoestrogens. Climacteric 2011; 14 (Suppl 2): 8-12.

5. Dębski R, Paszkowski T, Skrzypulec-Plinta V, et al. Polish Menopause and Andropause Society. https://e-medycyna.pl/baza-wiedzy/run,208publication,news,226, section, 1.html\#content.

6. Tice JA, Ettinger B, Ensrud K, et al. Phytoestrogen supplements for the treatment of hot flashes: The Isoflavone Clover Extract (ICE) Study: A randomized controlled trial. JAMA 2003; 290: 207-214.

7. Imhof M, Gocan A, Reithmayr F, et al. Effects of a red clover extract (MF11RCE) on endometrium and sex hormones in post-menopausal women. Maturitas 2006; 55: 76-81.

8. Geller SE, Shulman LP, van Breemen RB, et al. Safety and efficacy of black cohosh and red clover for the management of vasomotor symptoms: a randomized controlled trial. Menopause 2009; 16: 1156-1156.

9. Baber RJ, Templeman C, Morton T, et al. Randomized placebo-controlled trial of an isoflavone supplement and menopausal symptoms in women. Climacteric 1999; 2: 85-92.

10. Knight DC, Howes JB, Eden JA, Howes LG. Effects on menopausal symptoms and acceptability of isoflavone containing soy powder dietary supplementation. Climacteric 2001; 4: 13-18.

11. Kaari C, Haidar M, Junior J, et al. Randomized clinical trial comparing conjugated equine estrogens and isoflavones in postmenopausal women: a pilot study. Maturitas 2006; 53: 49-58.

12. Bicca ML, Horta BL, et al. Double-blind randomized clinical trial to assess the effectiveness of soy isoflavones in the relief of climacteric symptoms.

13. Levis S, Strickman-Stein N, Ganjei-Azar P, et al. Soy isoflavones in the prevention of menopausal bone loss and menopausal symptoms: a randomized, double-blind trial. Arch Intern Med 2011; 171: 1363-1369.

14. Fritz H, Seely D, Flower G, et al. Soy, red clover, and isoflavones and breast cancer: a systematic review. PLoS One 2013; 28: 8: e81968.

15. Colli MC, Bracht A, Soares AA, et al. Evaluation of the efficacy of flaxseed meal and flaxseed extract in reducing menopausal symptoms. Med Food 2012; 15: 840-845.

16. Shike M, Doane AS, Russo L, et al. The effects of soy supplementation on gene expression in breast cancer: a randomized placebo-controlled study. J Natl Cancer Inst 2014; 106: 1-12.

17. de Lemos ML. Effects of soy phytoestrogens genistein and daidzein on breast cancer growth. Ann Pharmacother 2001; 35: 1118-1121.

18. Frei-Kleiner S, Schaffner W, Rahlfs VW, et al. Cimicifuga racemosa dried ethanolic extract in menopausal disorders: a double-blind placebo-controlled clinical trial. Maturitas 2005; 51: 397-404.

19. Newton KM, Reed SD, LaCroix AZ, et al. Treatment of vasomotor symptoms of menopause with black cohosh, multibotanicals, soy, hormone therapy, or placebo: a randomized trial. Ann Intern Med 2006; 145: 869879.

20. Amsterdam JD, Yao Y, Mao JJ, et al. Randomized, double-blind, placebocontrolled trial of Cimicifuga racemosa (black cohosh) in women with anxiety disorder due to menopause. J Clin Psychopharmacol 2009; 29 : 478-483.

21. Jacobson JS, Troxel AB, Evans J, et al. Randomized trial of black cohosh for the treatment of hot flashes among women with a history of breast cancer. J Clin Oncol 2001; 19: 2739-2745.

22. Osmers R, Friede M, Liske E, et al. Efficacy and safety of isopropanolic black cohosh extract for climacteric symptoms. Obstet Gynecol 2005; 105: 1074-1083.

23. Wuttke W, Seidlová-Wuttke D, Gorkow C. The Cimicifuga preparation BNO 1055 vs. conjugated estrogens in a double-blind placebo-controlled study: effects on menopause symptoms and bone markers. Maturitas 2003; 44 Suppl 1: S67-S77.
24. Nappi RE, Malavasi B, Brundu B, Facchinetti F. Efficacy of Cimicifuga racemosa on climacteric complaints: a randomized study versus low-dose transdermal estradiol. Gynecol Endocrinol 2005; 20: 30-35.

25. Bai W, Henneicke-von Zepelin HH, Wang S, et al. Efficacy and tolerability of a medicinal product containing an isopropanolic black cohosh extract in Chinese women with menopausal symptoms: a randomized, double blind, parallel-controlled study versus tibolone. Maturitas 2007; 58: 31-41.

26. Borrelli F, Ernst E. Black cohosh (Cimicifuga racemosa): a systematic review of adverse events. Am J Obstet Gynecol 2008; 199: 455-466.

27. Roberts H, Safety of herbal medicinal products in women with breast cancer. Maturitas 2010; 66: 363-369.

28. Elia D, Mares P. Assessment of the tolerance and effectivenes of a food supplement Séréys ${ }^{\circledR}\left(\right.$ Femal $\left.{ }^{\circledR}\right)$ for menopausal women. Genesis 2008; 135: 12-15.

29. Winther K, Rein E, Hedman C. Femal, a herbal remedy made from pollen extracts, reduces hot flushes and improves quality of life in menopausal women: a randomized, placebo-controlled, parallel study. Climacteric 2005; 8: 162-170.

30. Kimura H, Gruber P. Perimenopausal symptoms such as hot flushes and moof swings are reduced by a standardized pollen and pistil extracts. Poster 2012. Available at: http://www.scicompdf.se/femal/gruber.pdf.

31. Sterns V, Johnson D, Rae JM, et al. Active tamoxifen metabolite plasma concentrations after coadministration of tamoxifen and the selective serotonin reuptake inhibitor paroxetine. J Natl Cancer Inst 2003; 95: 1758-1764.

32. Goldstein SR, Espié M, Druckmann R. Does purified Swedish pollen extract, a nonhormonal treatment for vasomotor symptoms, inhibit the CYP2D6 enzyme system? Menopause 2015; 22: 1212-1214. 